\title{
NORMAL RESPIRATORY AND CIRCULATORY PATHWAYS OF ADAPTATION IN EXERCISE ${ }^{1}$
}

\author{
By ROBERT A. BRUCE, ${ }^{2}$ FRANK W. LOVEJOY, JR.,2 RAYMOND PEARSON,2 \\ PAUL N. G. YU, ${ }^{2}$ GEORGE B. BROTHERS ${ }^{3}$ AND TULIO VELASQUEZ ${ }^{4}$ \\ (From the Chest Laboratory of the Department of Medicine of the University of Rochester \\ School of Medicine and Dentistry and the Medical Clinics of Strong Memorial and \\ Rochester Municipal Hospitals, Rochester, New York)
}

(Received for publication January 17, 1949)

Rahn and Otis (1) have described the normal changes in the alveolar gases during work and clemonstrated the quantitative relationships between alveolar oxygen, carbon dioxide, respiratory quotient and ventilation by means of continuous recording of gas analyses. They were able to delineate the pathways of change which are controlled by the excretion of carbon dioxide, and observe the effects of hyperpnea, hypoxia, hypoventilation and $\mathrm{CO}_{2}$ breathing as well. Pelnar (2), at first independently and later in conjunction with Rahn, observed the changes in expired air composition in normal subjects as well as in patients with cardiorespiratory impairment in relation to work. By means of a simple diagram relating the changes in $\mathrm{O}_{2}$ and $\mathrm{CO}_{2}$ content of expired air with the respiratory quotient during the stress of exercise, he felt that he could delineate respiratory function better than by any other means in a manner that paralleled the intensity of dyspnea in patients. Between the extremes of $a$ ) normal performance with a large circular curve and $b$ ) the abnormal performance with no change in these values during exercise, Pelnar found a series of results which were characteristic of the varied stages of dyspnea. Thus he was able to evaluate functional performance largely in terms of changes in the respiratory gases during exercise. In contrast to this are the older methods reported by Kaltreider and McCann (3) who relied chiefly on ventilation volumes and ventilation indices to appraise pulmonary capacity during work. Both Rahn and Otis (1) and Pelnar (2) express the

1 Aided by grants from the Hochstetter Fund, Lovejoy Fund, Fluid Research Fund, and the Rochester Gas and Electric Company Fund.

2 Bertha Hochstetter Buswell Research Fellow in Medicine.

3 Fellow of the General Education Board.

4 Rockefeller Visiting Fellow in Medicine. view that the level of ventilation is controlled in large measure by the $\mathrm{CO}_{2}$ when breathing normal ambient air in which the strong stimulus of hypoxia is lacking. The hypoxic stimulus of breathing $15 \%$ oxygen in normals or hypoxemia from pulmonary disease in patients clearly induces greater ventilation levels, however.

With these considerations in mind we have attempted to repeat these studies in both normal subjects and patients with disease to ascertain not only the normal respiratory pathways, but also the circulatory pathways during exercise and recovery. Thus the inter-relationships between the two can be considered in a way to demonstrate how the adaptations of each complement and spare the other. The techniques employed consist essentially of controlled work-loads by means of walking on a motor-driven treadmill and continuous gas analyses of expired air as well as determinations of electrocardiographic changes in rate and pattern, blood pressure and arterial oxygen saturation (oximeter) (Figure 1). Observations have not been limited to the basal state in order to obtain an appraisal of performance to a standardized stress of exercise in relation to ordinary circumstances in regard to diet, clothing and activity. In a separate report the statistical analyses of variability of normal performances under these conditions is presented (4). For the purpose of defining the normal pathways in this report, only the mean values of all pertinent observations obtained at minute intervals before, during, and after exercise are graphically considered in relation to each other.

\section{METHODS}

Thirty-five normal adults were used for this investigation (20 men, 15 women, ranging in age from 20 to 57 years). Each was allegedly healthy and gainfully employed, and none had any detectable sequelae of any previous cardiac or pulmonary disease. All rested before 


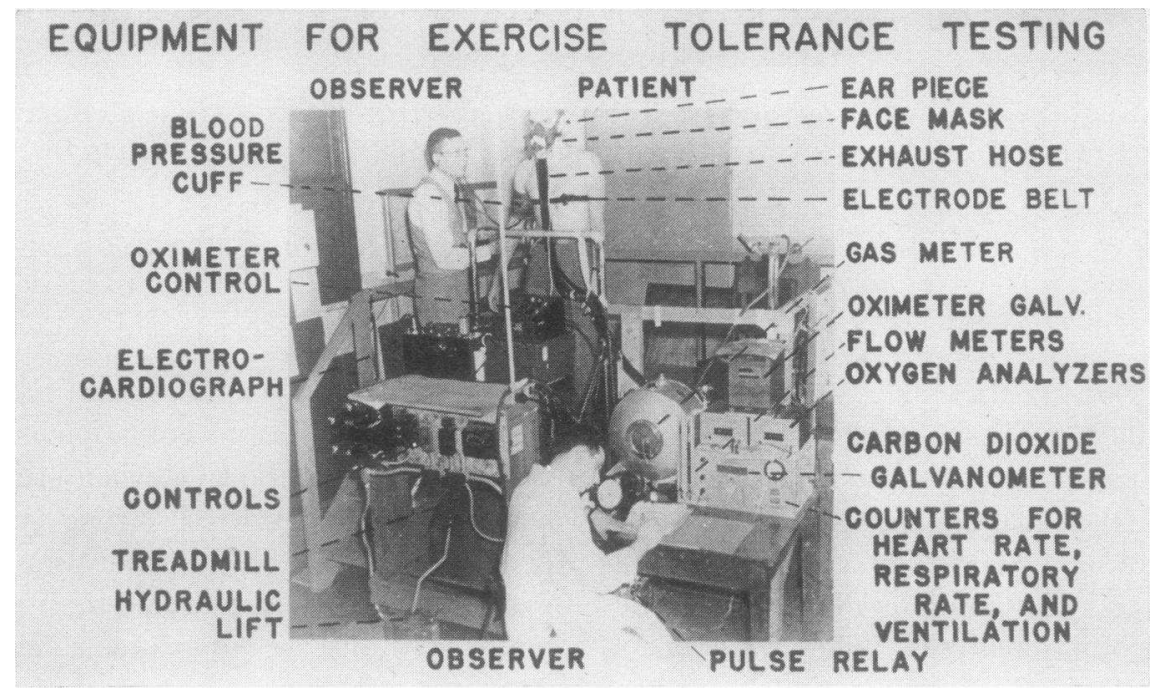

FIG. 1

Arrangement of equipment for exercise tolerance testing. One observer remains on the treadmill platform with the patient, records blood pressure, electrocardiogram, symptoms and signs. The other observer seated at the continuous analyzer records the heart rate, respiratory rate, ventilation volume, gas concentrations, and oximeter at one minute intervals.

the test period and then were observed during 10 minutes of rest sitting in a chair, 10 minutes level walking on the treadmill at the rate of $2.6 \mathrm{mph}(12 \mathrm{rpm}$ of the conveyor belt or 70 meters per minute velocity), and again for 10 minutes of recovery while sitting in a chair. Each was lightly clothed, and the barometric pressure and temperature were the prevailing room air values (747.3 \pm $1.1 \mathrm{~mm}$. $\mathrm{Hg}, 0.15 \%$ coefficient of variability) and $24.9 \pm$ $1.9^{\circ} \mathrm{C}(7.9 \%$ coefficient of variability).

The systemic blood pressure was obtained from an aneroid sphygmomanometer at frequent intervals, even during walking. Changes in arterial oxygen saturation, as well as variations in characteristics of the ear (green filter), were followed by an improved Millikan oximeter. Three electrodes attached to a rubber belt around the subject's chest for "ground," "indifferent" (right scapula) and "apical" contacts permitted registration of the chest lead electrocardiogram on a direct-writing instrument as well as continuous recording of the pulse from the amplified QRS spikes. Expired air gas data were obtained from a modified A-13A oxygen mask through a $11 / 2$ inch corrugated rubber hose mixing chamber conducting to a continuous gas analyzer. Ventilation volumes were electrically recorded from a low resistance (differential pressure less than 1 inch of water) wettest gasometer with accuracy of $\pm 1.65 \%$ at 100 liter per minute velocities. The respiratory rate was recorded by activation of a variable time-delay relay counter. Gas analyses were made continuously on aliquot (saturated with water) samples (at flow rates of 100$125 \mathrm{ml}$ per minute regulated by calibrated rotameters), by means of a thermal-conductivity cell for carbondioxide expressed in volumes per cent; and a suitable Pauling oxygen analyzer which determined the partial pressure of oxygen. The calibration of each was established and checked against room air prior to each test. The terminal portion of each expired breath (midcapacity air) ${ }^{5}$ was continuously sampled by the alternate pulsations of a very thin rubber balloon incorporated in the face piece of the mask for analyses of oxygen tension by a second Pauling analyzer and the derivation of changes in the physiological dead space. These values included the dead space of the mask which was reduced by careful packing with cotton. At the rates of flow stated above, the aliquot gas samples reached the analyzers after a lag of 10-20 seconds for expired air and 40-50 seconds for the mid-capacity air. Since the response of the $\mathrm{CO}_{2}$ analyzer was delayed to $99 \%$ peak reading in five minutes from zero concentration, acute changes in $\mathrm{CO}_{2}$ concentration could not be followed with accuracy. Hence no correction for inspired air volume from differences in nitrogen concentration in expired air were made, and estimates of the expired air respiratory quotients were only approximated values (averaging about $4 \%$ too high), (Figure 2 ).

5 In the traditional sense, alveolar air has the composition of that volume of air which is obtained at the end of forced expiration, hence includes in the last portion of the reserve air partition of the pulmonary capacity. "Midcapacity" air corresponds in gaseous composition to that obtained at the end of normal expiration. 
MAGNITUDE OF ERROR IN OXYGEN CONSUMPTION

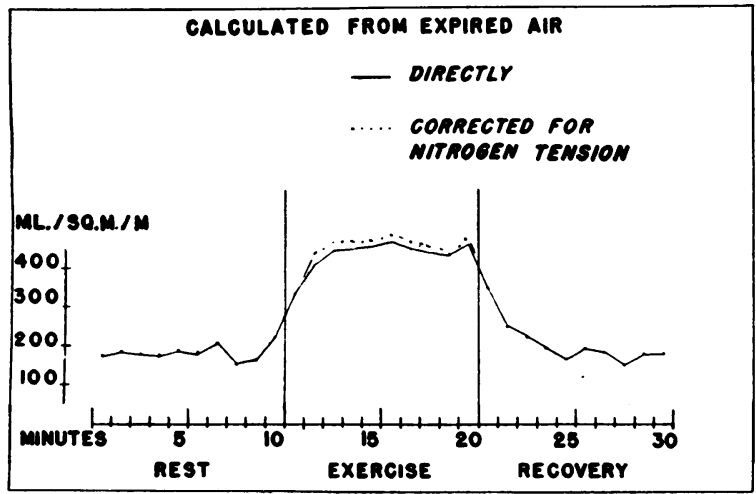

FIG. 2

The differences in oxygen consumption calculated directly are compared with those obtained by correction for changes in nitrogen tension. The magnitude of error by the short cut calculation is considered almost negligible in relation to the change in oxygen consumption initiated by exercise.

The reproducibility and accuracy of gas analyses by this method was estimated as variations of duplicate analyses from an ideal value of zero in terms of standard deviations as follows:

a) Haldane technique

b) Continuous analyzer

c) Between " $a$ " and " $b$ "

$\begin{array}{cc}\mathrm{CO}_{2} \text { vol. } \% & \mathrm{O}_{2} \text { vol. \% } \\ \pm 0.054 & \pm 0.058 \\ \pm 0.160 & \pm 0.028 \\ \pm 0.360 & \pm 0.0836\end{array}$

Consequently reliance was placed upon the oxygen values. Suitable conversion tables permitted rapid determination of the volumes per cent of oxygen absorbed (respiratory efficiency) from air from the observed expired air $\mathrm{pO}_{2}$ reading and the dry barometric pressure at the operating temperature. Similarly a factor was established for each subject that permitted conversion of the ventilation volumes observed into liters per square meter of body surface area at $760 \mathrm{~mm}$. $\mathrm{Hg}$ and $0^{\circ} \mathrm{C}$ dry. The oxygen consumption was estimated from the product of the corrected minute ventilation and the respiratory efficiency and ranged between 96-101\% (depending on the R.Q.) of the true value based upon corrections for nitrogen differences in inspired and expired air. All primary observations were recorded and graphed at one minute intervals (Figure 3). From these data, average values per period, dead space volumes, oxygen debt, ventilation indices, etc. were calculated.

Accessory procedures routinely employed were the determination of maximum mask breathing capacity by means of voluntary hyperventilation for 30 seconds at the completion of the above test, changes in oximeter

6 The standard error for oxygen analyses determined by the Haldane technique was 3.3 times greater than that obtained by the Pauling oxygen analyzer. readings with breath-holding, and estimation of lung-toear circulation time with the oximeter.

\section{RESULTS}

Inasmuch as statistical analyses showed no significant differences in the data according to the sex of the subjects, all the consecutive one minute observations for the mean values of both sexes have been compiled together. The resting values (allowing for differences in basal state, tempera-

\section{EXERCISE PERFORMANCE (MORmal male)}

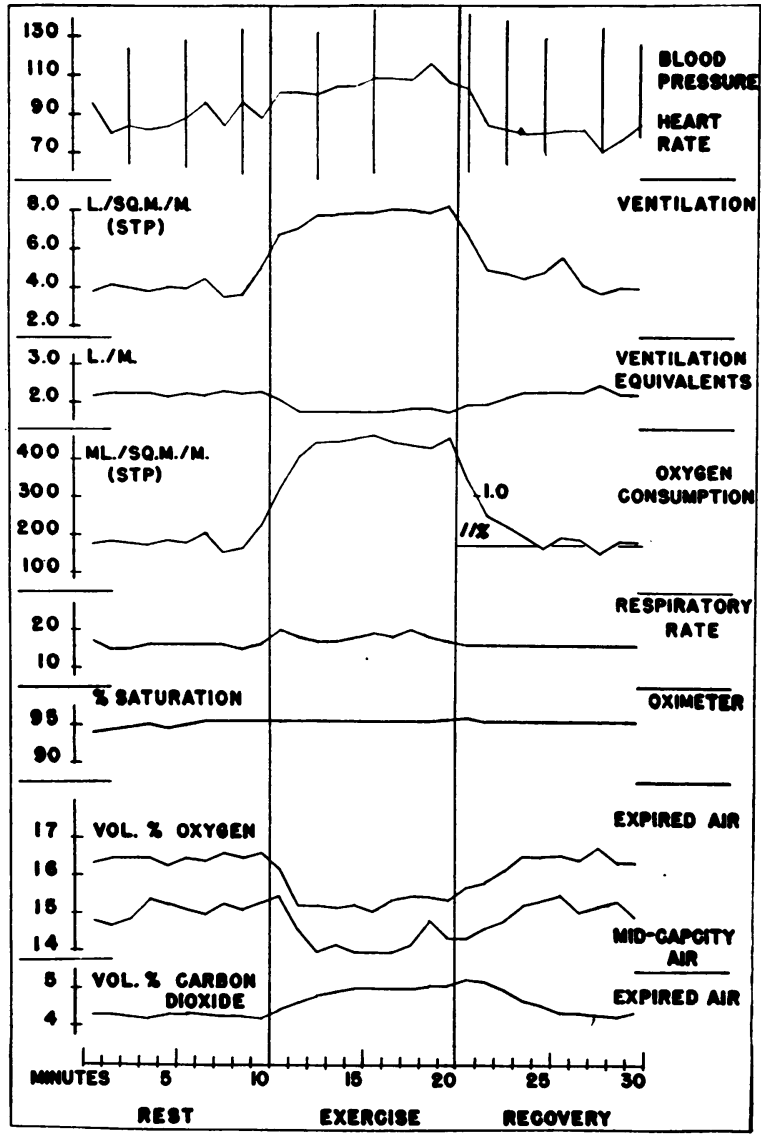

FIG. 3

Typical exercise performance graph of a normal male subject. The values of " $11 \%$ " and " 1.0 " adjacent to the recovery curve for oxygen consumption refer to oxygen debt and half-time recovery time, respectively. The value of minute-by-minute observations of multiple measurements is illustrated by the fleeting changes in heart rate, ventilation, and oxygen consumption occurring on the seventh, 19th, and 28th minutes. These variations were involuntary reactions to environmental stimuli initiated by visitors during the period of observation. The subject was unaware of these variations. 
RATES OF ADAPTATION

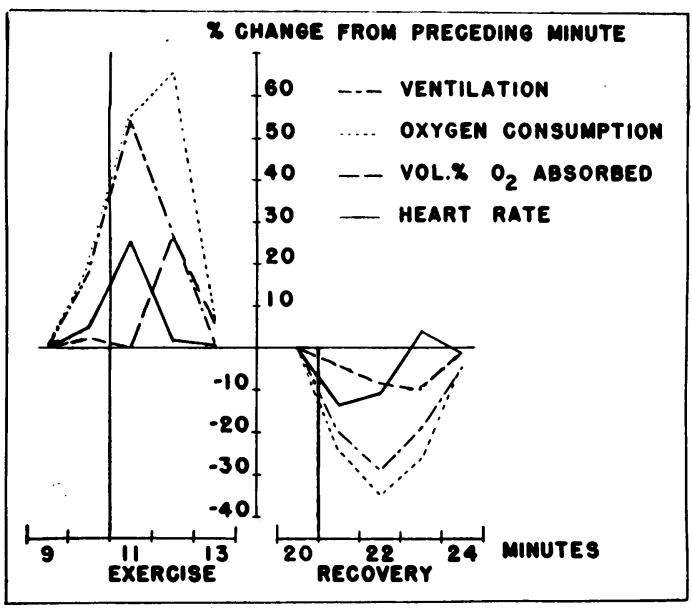

FIG. 4

The rates of adaptation to changing states of activity are compared on the basis of per cent change from the preceding mean one minute values for ventilation volume, oxygen consumption, volumes per cent of oxygen absorbed from expired air and heart rate for the first four minutes of exercise and recovery. The 10 th minute represents changes from standing. Note that ventilation and oxygen consumption show the greater changes first, and that the change in volumes per cent of oxygen absorbed is delayed, causing the final increment in oxygen consumption during exercise.

ture and pressure) are in accord with those reported by others.

The rates of changes in the observations of ventilation, oxygen consumption, respiratory efficiency (volumes per cent of oxygen absorbed) and the heart rate are shown in Figure 4. These changes are expressed as percentage changes in the several factors from the immediately preceding minute during the periods of adaptation to and from a steady state of exercise. The values for the 10th minute of observation represent the changes induced by standing up and are of smaller magnitude than those for walking. As a result of exercise both ventilation and heart rate promptly rise, but the greatest change is confined to the first minute. The oxygen consumption initially rises in proportion to the ventilatory response, but thereafter it continues to rise during the next minute because of the greater respiratory efficiency. By the 13th minute of observation all factors have approached a new and relatively stable range during the steady state of exercise. In recovery the reverse changes promptly occur, but in this instance there are two apparent differences: $a$ ) there is no lag in decline of respiratory efficiency, and $b$ ) the heart rate is restored toward the resting level more rapidly than the other factors.

There are changes in the physiological dead space along with the increments in the mid-capacity vontilation associated with exercise (Figure 5). The latter shows an almost linear rise during the first three minutes of exercise as well as a linear decline in three minutes during recovery from exercise. During the last seven minutes of exercise there is a just perceptible increase in the mid-capacity ventilation. The slight discrepancies between total ventilation and midcapacity ventilation appear to be related to fluctuations in the physiological dead space. On the average, throughout the three periods of observation the dead space represents $21 \%$ of the tidal volume. Both the relative and absolute values for the dead space are lower than those reported by others (about 26 to $30 \%[5,6]$ ), and recent improvements in the face mask sampling device yield somewhat higher dead space values indicating a technical error as partly responsible for these discrepancies. Although the dead space shows a

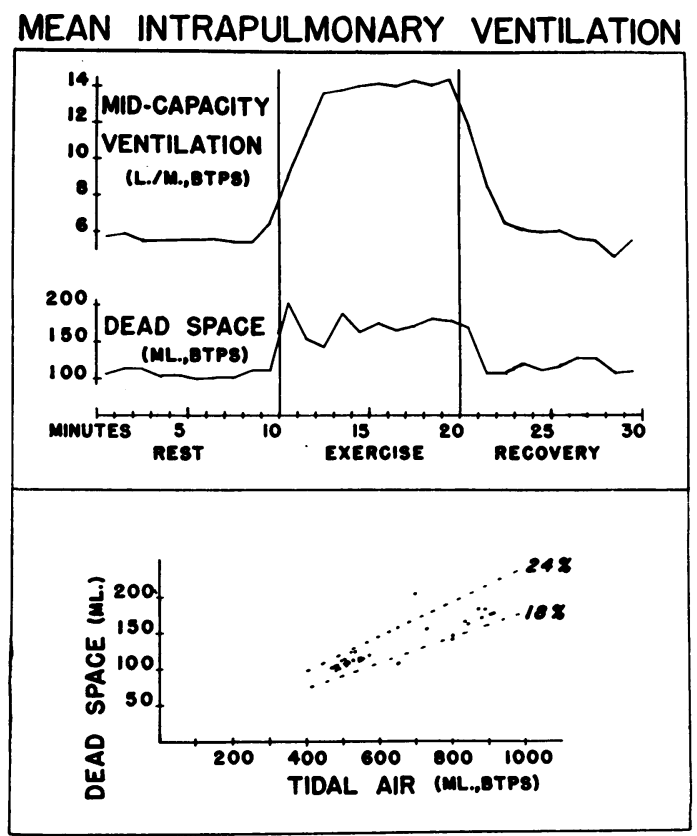

FIG. 5

Relationships of mean intrapulmonary ventilation, physiological dead space, and tidal air volume in ambient values, B. T. P. S., in normal subjects. 
MEAN RESPIRATORY PATHWAYS

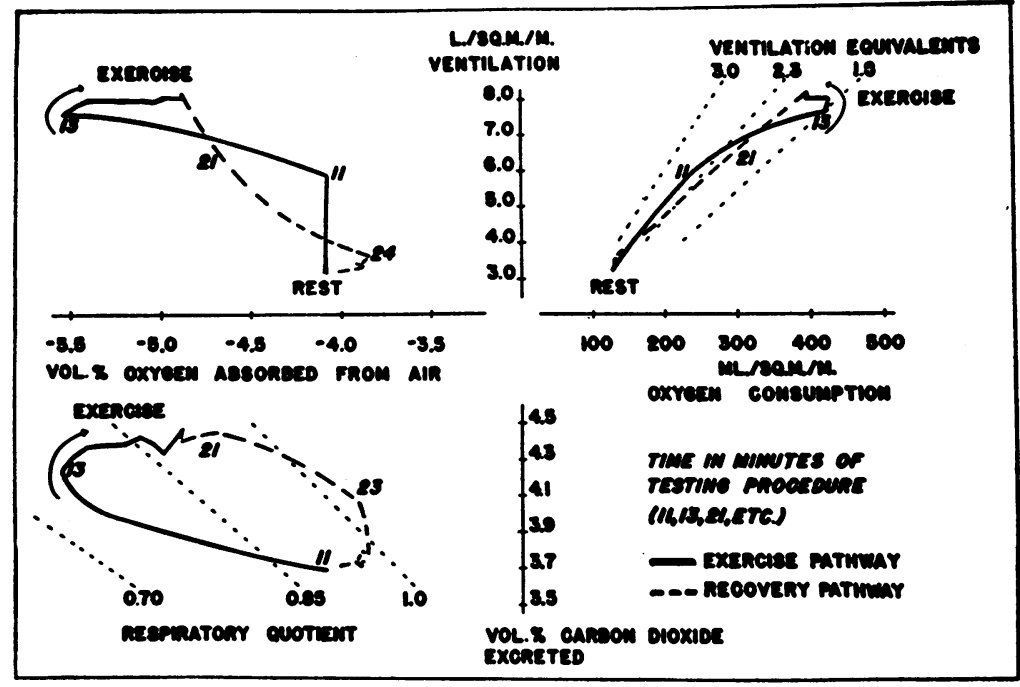

Fig. 6

The mean respiratory pathways in normal subjects illustrate the various inter-relations between ventilation, oxygen consumption, ventilation equivalents for oxygen, respiratory quotient, and changes in activity from rest to exercise and back to rest again. The arrows indicate the direction of the loops, and the numbers adjacent to the loops indicate the specific minutes of observation during the testing procedure. The iso-R.Q. lines are indicated by the background grid; note that the ultimate exercise R.Q. is slightly lower than the resting value. (Inasmuch as the oxygen consumption and dead space change with activity, iso-ventilation lines cannot be applied to this graph). Note that the ventilation equivalent for oxygen is lower during exercise than at rest, indicating increased pulmonary capillary flow and more effective oxygenation of the pulmonary arterial blood. See text for further discussion.

disproportionately large value early in exercise followed by a transient decline, the average dead space value during exercise is $62 \%$ larger than the resting values.

The mean respiratory pathways of adaptation from a steady state of rest to that of exercise and the converse during recovery are shown in Figure 6. The oxygen consumption at first increases proportionately with the increments in ventilation, but a little later it rises more rapidly because of greater respiratory efficiency (volumes per cent of oxygen absorbed, or reciprocally, diminishing ventilation equivalents for oxygen). The improved respiratory efficiency is a function of more effective alveolar ventilation and circulation as well as alterations in blood flow and composition (see below). Concurrently the physiological dead space enlarges. After the 13th minute of observation (third minute of exercise), the estimated oxygen consumption gradually declines $5 \%$ as the respiratory efficiency slowly recedes (Figure 6) with a perceptible rise in mid-capacity ventilation (Figure 5). On the average there is a $22 \%$ increase in respiratory efficiency at this level of work. The estimated R.Q. of expired air shows wide fluctuations during the adaptive phases because of the differences in rates of change in the several factors representing ventilation and circulation. The direction of change in the R.Q. is always a clockwise loop showing a low value early in exercise as the highest values for respiratory efficiency are achieved by the 13th minute of observation (Figure 6). During steady states of performance, either rest or activity, the expired air R.Q. may be considered representative of tissue metabolism throughout the body. With this level of work, the R.Q. does not rise above the resting value as it does with more severe levels of work 


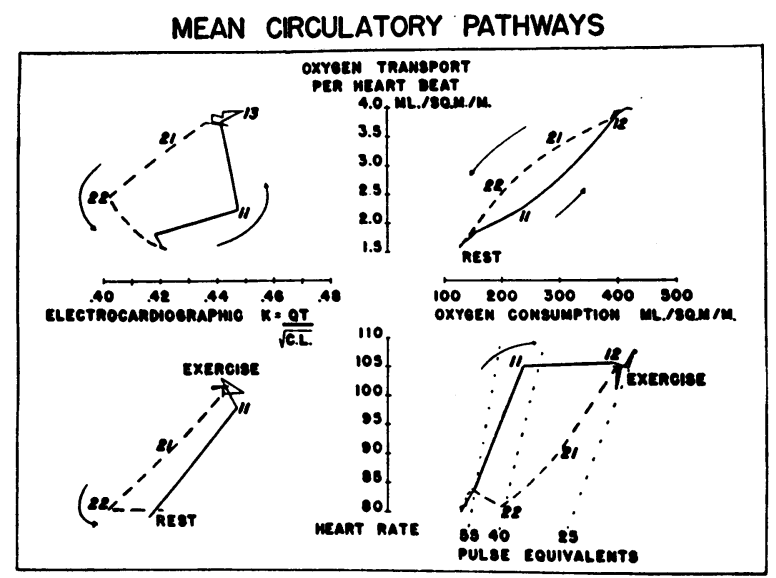

FIG. 7

The mean circulatory pathways in normal subjects illustrate the various inter-relations of heart rate, oxygen consumption, pulse equivalents for oxygen, amount of oxygen carried per heart beat and electrocardiographic $\mathrm{K}$ constant during changes in activity from rest to exercise, and back to rest again. Note that the heart rate rises rapidly during the first minute, and maintains a plateau thereafter during exercise. The decline in pulse equivalents indicates a rise in stroke volume. The arrows indicate the direction of the loops, and the numbers adjacent to the loops indicate the specific minutes of observation during the testing procedure.

(7). With the onset of recovery the R.Q. of expired air transiently exceeds 1.0 as retained $\mathrm{CO}_{2}$ continues to be excreted in excess of the rate of oxygen absorption reduced with diminished tissue demands for oxygen. ${ }^{7}$ Simultaneously the level of ventilation is maintained above the tissue demands by the stimulus of retained $\mathrm{CO}_{2}$, and concurrently most of the aerobic oxygen debt is repaid.

The mean circulatory pathways, based upon consecutive observations of minute heart rate, oxygen consumption, oxygen transport per heart beat, and systolic time are shown in Figure 7 . The systolic time is derived from the (chest lead)

7 Pelnar utilizes the abnormally high R.Q. values during steady states of either rest or moderate activity to recognize the presence of hyperventilation, beyond the metabolic needs of the tissues, caused by neuropsychogenic factors. The usage of the term "respiratory quotient" in this sense may be unfortunate, especially when consideration is given to the limitations in accurately measuring the concentration of carbon dioxide by the present authors. Possibly the term "respiratory ratio" or ratio of carbon dioxide excreted to oxygen absorbed would be more appropriate.

electrocardiographic changes in the $\mathrm{K}$ constant, or observed $\mathrm{QT}$ interval divided by the square root of the cycle length to correct for changes due to rate. The oxygen transport is derived from the following equations :

(a)

$$
\begin{aligned}
& \text { Cardiac Output } \\
& \quad=\text { Heart rate } \times \text { stroke volume } \\
& \quad=\frac{\text { Oxygen consumption }}{\mathrm{A}-\mathrm{V} \mathrm{O}_{2} \text { difference }}
\end{aligned}
$$

and by substitution:

\section{(b)}

$$
\begin{aligned}
& \text { Oxygen Transport } \\
& =\frac{\text { Oxygen consumption }}{\text { Heart rate }} \\
& =\text { Stroke volume } \times \mathrm{A}-\mathrm{V} \mathrm{O}_{2} \text { difference. }
\end{aligned}
$$

The oxygen transport also represents the average rate of oxygen transfer from either alveolar air to mixed venous blood, or arterial blood to peripheral tissues, per unit of physiological time of one heart beat. Statistical analyses of rates of change in large series of observations on normal subjects show that there is a $7 \%$ increase in cardiac output for every $10 \%$ increment in oxygen consumption, and that the stroke volume shows somewhat greater increases than the $\mathrm{A}-\mathrm{V}$ oxygen difference with increasing cardiac output (8). Similarly analyses of the present series of mean values shows a linear relationship of a high order of significance between oxygen consumption and oxygen transport $(r=0.938)$. With the initiation of exercise the heart rate promptly accelerates during the first minute of exercise and thereafter remains on a plateau (Figure 4). Since the oxygen consumption continues to rise, and hence the cardiac output, further increases in cardiac output must be achieved by larger stroke volumes. The rates of change in oxygen transport show the greatest increase during the second minute of exercise when the $\mathrm{A}-\mathrm{V}$ oxygen difference probably is also widening due to a reduction of mixed venous oxygen content caused by blood returning from the exercising muscles (7). Concurrently the respiratory efficiency is attaining its highest and the R.Q. its lowest values. During these adaptive changes in circulation (Figure 3 ) there are corresponding changes in the systolic time manifested by the electrocardiographic $\mathrm{K}$ constant. Initially with exercise there is an appreciable rise during the first minute followed by gradual fluctuations dur- 
ing the remainder of exercise; on the average the exercise systolic time is $7 \%$ greater than that observed during the resting period. With recovery the $\mathrm{K}$ value falls during the 21 st and 22 nd minute of observation to $9 \%$ below the exercise range and then gradually resumes the resting value. In none of the normal subjects studied at this level of work were there any significant alterations of the QRS complexes, ST segments, or $\mathrm{T}$ waves, other than the effects of a more rapid rate; i.e., no changes in the electrocardiogram that could be associated with possible or probable insufficiency of the coronary circulation.

\section{DISCUSSION}

The continuous recording of the heart rate electrocardiographically, ventilation volume, and respiratory efficiency of expired and mid-capacity air, as well as the carbon dioxide concentration during changes in the metabolic demand initiated by exercise greatly supplements the existing information about either ventilatory performance or alterations in gaseous composition. By these means the pathways of respiratory and circulatory adaptation to the stress of exercise can be observed in terms of degrees, as well as rates, of change in a manner that demonstrates the complementary sparing action of the several variables. Appreciable uniformity of results can be approximated by control of the work-load in proportion to the body size by means of walking on a motordriven treadmill. These studies have not been performed under basal conditions in order to observe the responses in both normal subjects and patients with cardio-respiratory diseases under ordinary circumstances of daily activity. An analysis of the range of variability and reproducibility of results in normals and alterations presented by patients with disease will be the subject of later reports. The resting values observed by these methods are in reasonable accord with those observed by others employing the TissotHaldane methods (9). The pathways of responses for expired air are similar to the findings of Rahn and Otis (1) for normal alveolar pathways in exercise as well as those of Pelnar (2) for the changes in expired air composition initiated by exercise.

These studies demonstrated the following changes which regularly occur with exercise: ventilation and heart rate promptly rise to a higher plateau, but the heart rate levels off by the second minute of exercise whereas the mid-capacity ventilation continues to increase in a linear fashion for three minutes. The R.Q. of expired air initially declines with exercise as the respiratory efficiency (denominator, or volumes per cent of oxygen absorbed) rises and overshoots the steady state value by the third minute of exercise (Figure 6). This transient change is an expression of greater alveolar ventilation and circulation (together with enlarged physiological dead space) as well as greater diffusion of oxygen into more rapidly circulating blood which has a lower content of oxygen as a result of increased muscular demand in exercise ( 7$)$. The arterial oxygen saturation is maintained; hence the $A-V$ oxygen difference widens; and since the oxygen consumption continues to rise for three minutes (and heart rate does not), there is a progressive increase in stroke volume. The striking increase in oxygen transport per heart beat during the second minute of exercise is an expression of the changes in both the $\mathrm{A}-\mathrm{V}$ oxygen difference and the stroke volume since it is the product of these two factors. As the apparent circulatory lag in adaptation to exercise is corrected, the expired air R.Q. gradually rises during the further attainment of the steady state of exercise and the respiratory efficiency slowly declines; ventilation just perceptibly rises; and oxygen consumption diminishes by about $6 \%$. These changes are considered to reflect peripheral circulatory adjustments as vasodilatation occurs to dissipate heat and slight arteriolization of venous blood from small peripheral shunts permits the mixed venous oxygen content to rise (and narrow the $\mathrm{A}-\mathrm{V}$ oxygen difference) $(10,11)$. With these changes there is no significant change in diastolic blood pressure although the systolic pressure, and hence, the pulse pressure increase. The alterations in the systolic time expressed by the electrocardiographic $\mathrm{K}$ constant are considered expressions of changes in blood flow as well as contractile force and ventricular emptying associated with increased stroke volume. Some individuals exhibit slight reductions in both heart rate and ventilation about the seventh minute of exercise, indicating further adjustments in performance; possibly these changes account for the phenomenon of the "second wind" 
subjectively observed at more severe levels of work.

Recovery from exercise begins at once, and in normal subjects at this level of work it is largely completed within three minutes. The first variable to approach the resting level is the heart rate which corroborates the usefulness of the recovery pulse as a measure of physical fitness (12). During this time the expired air R.Q. rises above 1.0 as retained carbon dioxide continues to be excreted, delaying somewhat the restoration of ventilation to the resting level although favoring the repayment of most of the aerobic oxygen debt.

\section{SUMMARY}

1. The mean respiratory and circulatory pathways of adaptation to the stress of exercise have been studied in 35 normal adults. The work-load was regulated by treadmill walking, and multiple observations were made continuously at one minute intervals before, during, and after the exercise.

2. The previously reported changes in gas composition were confirmed.

3. The additional values of simultaneous consideration of circulatory changes are discussed.

4. The adaptive changes in respiration and circulation are shown to complement and spare each other by the differences in time and rate of change.

\section{ACKNOWLEDGMENT}

The authors wish to acknowledge the helpful criticisms of Doctors W. S. McCann and N. L. Kaltreider of the Department of Medicine of the University of Rochester in the editing of this paper.

\section{BIBLIOGRAPHY}

1. Rahn, H., and Otis, A. B., Continuous analysis of alveolar gas composition during work, hyperpnea, hypercapnia, and hypoxia. J. Appl. Physiol., 1949, 1, 717.

2. Pelnar, P., A new method of examining the efficiency of the respiratory and circulatory system. Nádiladem Česhé Akademie Věd A Uměni, V Praze, 1948.

3. Kaltreider, N. L., and McCann, W. S., Respiratory response during exercise in pulmonary fibrosis and emphysema. J. Clin. Invest., 1937, 16, 23.

4. Bruce, R. A., Pearson, R., Lovejoy, F. W., Yu, P. N. G., and Brothers, G. B., Variability of respiratory and circulatory performance during standardized exercise. J. Clin. Invest., 1949, 28, 1431.

5. Hurtado, A., Fray, W. W., Kaltreider, N. L., and Brooks, W. D. W., Studies of total pulmonary capacity and its subdivisions. V. Normal values in female subjects. J. Clin. Invest., 1934, 13, 169.

6. Fowler, W. S., Lung function studies. II. Respiratory dead space. Am. J. Physiol., 1948, 154, 405.

7. Robinson, S., Experimental studies of physical fitness in relation to age. Arbeitsphysiol., 1938, 10, 251.

8. Pearson, R., and Bruce, R. A., Unpublished studies, 1949.

9. Baldwin, E. de F., Cournand, A., and Richards, D. W., Pulmonary insufficiency. I. Physiological classification, clinical methods of analysis, standard values in normal subjects. Medicine, 1948, 27, 243.

10. Dill, D. B., The economy of muscular exercise. Physiol. Rev., 1936, 16, 263.

11. Grant, R. T., and Bland, E. F., Observations on arterio-venous anastomoses in the human skin and in the bird's foot with special reference to the reaction to cold. Heart, 1931, 15, 385.

12. Johnson, R. E., Brouha, L., and Darling, R. C., Test of physical fitness for strenuous exertion. Rev. Canad. de biol., 1942, 1, 491. 